\title{
Crystallochemistry of defects in lead telluride films
}

\author{
D.M. Freik, M.A. Ruvinskii, B.M. Ruvinskii, M.A. Galushchak \\ Physico-chemical institute at Precarpathian university named by V.Stefanyk, \\ Shevchenko Str., 57, Ivano-Frankivsk, 76000, Ukraine.
}

\begin{abstract}
The crystallochemical model of vapor-phase epitaxy of the lead telluride films has been proposed with the supposition about the simultaneous formation of singly charged, doubly charged and electroneutral Frenkel defects in the cationic sublattice. It has been shown that in spite of the large concentration of partly compensated doubly charged defects the singly charged Frenkel pairs $\mathrm{Pb}_{i}^{+}$and $V_{\mathrm{Pb}}^{-}$also play an essential role in change carrier concentration in PbTe films. The results of numerical calculation agree with the available experimental data of the dependence of charge carrier's concentration in films upon the partial pressure of tellurium vapor and the deposition's temperature in the hot-wall method.
\end{abstract}

Keywords: crystallochemistry, defects, films, lead telluride.

Paper received 14.09.00; revised manuscript received 10.01.01; accepted for publication 16.02.01.

1. It is known that it is possible to control the electric properties (i.e. conduction type and charge carrier concentration) of lead chalcogenides by varying their composition within the homogeneity region. However, there is no agreement in the literature concerning the predominant type of intrinsic defects in $\mathrm{A}^{\mathrm{IV}} \mathrm{B}^{\mathrm{VI}}$ and their charge state. For instance, based on analysis of the state diagrams and processes of annealing of lead telluride, the authors of [3] assumed the existence of singly charged acceptors $V_{\mathrm{Pb}}^{-}$. Conclusions about the predominance of disordering in metal sublattice with formation of singly charge defects $\mathrm{Pb}_{i}^{+}$and $V_{\mathrm{Pb}}^{-}$, regardless of enrichment by lead or chalcogenide, are drawn in [4]. There are also various opinions concerning the charge state of interstitial lead [5]. While in [6] it is assumed to be the doubly ionized donor $\left(\mathrm{Pb}_{i}^{2+}\right)$, in [3] it is considered as singly ionized $\mathrm{Pb}_{i}^{+}$. There have been attempts to explain the experimental dependence of electrical parameters of $\mathrm{PbTe}$ thin films on growth conditions by formation of singly charged interstitial atoms $\mathrm{Pb}_{i}^{+}$and doubly charged vacancies $V_{\mathrm{Pb}}^{2-}$. In calculations of energy spectrum of atomic defects, their electrical activity is explained within the model assuming doubly charged metal $V_{\mathrm{Pb}}^{2-}$ and chalcogen $V_{\mathrm{Te}}^{2+}$ vacancies [5]. In [8] it has been shown that at low temperatures and high chalcogen vapour pressure, PbTe crystals have significant number of neutral intrinsic point defects along with singly ionized defects.

2. For the first time we introduce the model of metal sublattice disordering in PbTe thin films according to Frenkel mechanism with simultaneous formation of singly and doubly charged, as well as neutral defects: inter- stitial lead atoms $\mathrm{Pb}_{i}^{+}, \mathrm{Pb}_{i}^{2+}, \mathrm{Pb}_{i}^{0}$ and lead vacancies $V_{\mathrm{Pb}}^{-}, V_{\mathrm{Pb}}^{2-}, V_{\mathrm{Pb}}^{0}$.

The equilibrium of «bulk weight - vapour» and «vapour-condensate» systems in the process of film growth from the vapour phase by the hot-wall method can be described by the following system of quasi-chemical reactions [10]:

$$
\begin{aligned}
& \mathrm{PbTe}^{S} \leftrightarrow \mathrm{Pb}^{V}+1 / 2 \mathrm{Te}_{2}^{V}, K_{\mathrm{PbTe}}\left(T_{e}\right)=P_{\mathrm{Pb}} \cdot P_{\mathrm{Te}_{2}}^{1 / 2} \\
& \mathrm{~Pb}^{V} \leftrightarrow \mathrm{Pb}_{i}^{+}+e^{-}, K_{\mathrm{Pb}, V}^{\prime}\left(T_{s}\right)=\left[\mathrm{Pb}_{i}^{+}\right] \cdot n \cdot P_{\mathrm{Pb}}^{-1} \\
& 1 / 2 \mathrm{Te}_{2}^{V} \leftrightarrow V_{\mathrm{Pb}}^{-}+\mathrm{Te}_{\mathrm{Te}}^{0}+h^{+}, \\
& K_{\mathrm{Te}_{2}, V}^{\prime}\left(T_{s}\right)=\left[V_{\mathrm{Pb}}^{-}\right] \cdot p \cdot P_{\mathrm{Te}_{2}}^{-1 / 2} \\
& \mathrm{~Pb}^{V} \leftrightarrow \mathrm{Pb}_{i}^{2+}+2 e^{-}, K_{\mathrm{Pb}, V}^{\prime \prime}\left(T_{s}\right)=\left[\mathrm{Pb}_{i}^{2+}\right] \cdot n^{2} \cdot P_{\mathrm{Pb}}^{-1} \\
& 1 / 2 \mathrm{Te}_{2}^{V} \leftrightarrow V_{\mathrm{Pb}}^{2-}+T e_{\mathrm{Te}}^{0}+2 h^{+}, \\
& K_{\mathrm{Te}_{2}, V}^{\prime \prime}\left(T_{s}\right)=\left[V_{\mathrm{Pb}}^{2-}\right] \cdot p^{2} \cdot P_{\mathrm{Te}_{2}}^{-1 / 2} .
\end{aligned}
$$

Here equation (1) determines the equilibrium of «bulk weight - vapour» system in the process of PbTe decomposition in the evaporator at temperature $T_{e}$. Reactions (2)-(5) determine the equilibrium in the system «vapourcondensate» that is responsible for the intrinsic atomic defects at precipitation (substrate) temperature $T_{s}$.

The intrinsic conductivity and ionization of Frenkel defects are described by equations (6) and (7)-(12), respectively: 


$$
\begin{aligned}
& \ll 0 »=e^{-}+h^{+}, \quad K_{i}\left(T_{s}\right)=n \cdot p ; \\
& \ll 0 »=V_{\mathrm{Pb}}^{-}+\mathrm{Pb}_{i}^{+}, \quad K_{F}^{\prime}\left(T_{s}\right)=\left[V_{\mathrm{Pb}}^{-}\right]\left[\mathrm{Pb}_{i}^{+}\right] ; \\
& \ll 0 »=V_{\mathrm{Pb}}^{2-}+\mathrm{Pb}_{i}^{2+}, \quad K_{F}^{\prime \prime}\left(T_{s}\right)=\left[V_{\mathrm{Pb}}^{2-}\right]\left[\mathrm{Pb}_{i}^{2+}\right] ; \\
& \ll 0 »=V_{\mathrm{Pb}}^{-}+\mathrm{Pb}_{i}^{2+}+e^{-}, \quad K_{F}^{(e)}\left(T_{s}\right)=\left[V_{\mathrm{Pb}}^{-}\right]\left[\mathrm{Pb}_{i}^{2+}\right] n ; \\
& \ll 0 »=V_{\mathrm{Pb}}^{2-}+\mathrm{Pb}_{i}^{+}+h^{+}, K_{F}^{(h)}\left(T_{s}\right)=\left[V_{\mathrm{Pb}}^{2-}\right]\left[\mathrm{Pb}_{i}^{+}\right] p ; \\
& \mathrm{Pb}_{i}^{0} \leftrightarrow \mathrm{Pb}_{i}^{+}+e^{-}, \quad K_{a}\left(T_{s}\right)=\left[\mathrm{Pb}_{i}^{+}\right] n /\left[\mathrm{Pb}_{i}^{0}\right] ; \\
& V_{\mathrm{Pb}}^{0} \leftrightarrow V_{\mathrm{Pb}}^{-}+h^{+}, \quad K_{b}\left(T_{s}\right)=\left[V_{\mathrm{Pb}}^{-}\right] p /\left[V_{\mathrm{Pb}}^{0}\right] ; \\
& \mathrm{Pb}_{i}^{0} \leftrightarrow \mathrm{Pb}_{i}^{2+}+2 e^{-}, \quad K_{a}^{\prime}\left(T_{s}\right)=\left[\mathrm{Pb}_{i}^{2+}\right] n^{2} /\left[\mathrm{Pb}_{i}^{0}\right] ; \\
& V_{\mathrm{Pb}}^{0} \leftrightarrow V_{\mathrm{Pb}}^{2-}+2 h^{+}, \quad K_{b}^{\prime}\left(T_{s}\right)=\left[V_{\mathrm{Pb}}^{2-}\right] p^{2} /\left[V_{\mathrm{Pb}}^{0}\right] .
\end{aligned}
$$

The general electroneutrality condition is:

$$
p+\left[\mathrm{Pb}_{i}^{+}\right]+2\left[\mathrm{~Pb}_{i}^{2+}\right]=n+\left[V_{\mathrm{Pb}}^{-}\right]+2\left[V_{\mathrm{Pb}}^{2-}\right] \text {. }
$$

From (1)-(15) we obtain the equation determining the charge carriers concentration $(n)$ in terms of quasi-chemical reaction constants $K$ and $P_{\mathrm{Te}_{2}}$ :

$$
A n^{4}+B n^{3}-C n-D=0 \text {. }
$$

Here

$$
\begin{aligned}
& A=2 K_{\mathrm{Te}_{2}, V}^{\prime \prime} K_{F}^{\prime} \cdot P_{\mathrm{Te}_{2}}^{1 / 2} \cdot\left(K_{\mathrm{PbTe}} K_{\mathrm{Pb}, V}^{\prime} K_{\mathrm{Te}_{2}, V}^{\prime} K_{i}\right)^{-1} ; \\
& B=1+K_{F}^{\prime} \cdot P_{\mathrm{Te}_{2}}^{1 / 2} \cdot\left(K_{\mathrm{PbTe}}^{\prime} K_{P b, V}^{\prime}\right)^{-1} \\
& C=K_{i}+K_{\mathrm{PbTe}} K_{\mathrm{Pb}, V}^{\prime} \cdot P_{\mathrm{Te}_{2}}^{-1 / 2} ; \\
& D=2 K_{\mathrm{PbTe}} K_{\mathrm{Pb}, V}^{\prime \prime} \cdot P_{\mathrm{Te}_{2}}^{-1 / 2} .
\end{aligned}
$$

The charge carrier concentration is determined experimentally by Hall effect:

$$
n_{H}=n-p=n-K_{i} \cdot n^{-1} \text {. }
$$

Within the suggested model, it is also possible to determine the concentrations of charged and electroneutral defects:

$$
\begin{aligned}
& {\left[\mathrm{Pb}_{i}^{+}\right]=K_{\mathrm{PbTe}} K_{\mathrm{Pb}, V}^{\prime} n^{-1} P_{\mathrm{Te}_{2}}^{-1 / 2} ;} \\
& {\left[\mathrm{Pb}_{i}^{2+}\right]=K_{\mathrm{PbTe}} K_{\mathrm{Pb}, V}^{\prime \prime} n^{-2} P_{\mathrm{Te}_{2}}^{-1 / 2} ;} \\
& {\left[V_{\mathrm{Pb}}^{-}\right]=K_{F}^{\prime} \cdot n \cdot P_{\mathrm{Te} 2}^{1 / 2}\left(K_{\mathrm{PbTe}} K_{\mathrm{Pb}, V}^{\prime}\right)^{-1} ;} \\
& {\left[V_{\mathrm{Pb}}^{2-}\right]=K_{\mathrm{Te}_{2}, V}^{\prime \prime} K_{F}^{\prime} \cdot n^{2} \cdot P_{\mathrm{Te}}^{1 / 2}\left(K_{\mathrm{PbTe}} K_{\mathrm{Pb}, V}^{\prime} K_{\mathrm{Te}_{2}, V}^{\prime} K_{i}\right)^{-1} ;} \\
& {\left[\mathrm{Pb}_{i}^{0}\right]=K_{\mathrm{PbTe}} K_{\mathrm{Pb}, V}^{\prime} K_{a}^{-1} P_{\mathrm{Te}}^{-1 / 2} ;} \\
& {\left[V_{\mathrm{Pb}}^{0}\right]=K_{F}^{\prime} K_{i} P_{T_{2}}^{1 / 2}\left(K_{\mathrm{PbTe}} K_{\mathrm{Pb}, V}^{\prime} K_{b}\right)^{-1} .}
\end{aligned}
$$

3. The intrinsic conductivity constant $K_{i}$ in equation (6) was determined from the temperature dependencies of the band-gap $E_{g}(T)$ and effective mass $m(T)$ [11]:
$K_{i}\left(T_{s}\right)=N_{c} \cdot N_{V} \cdot e^{-E g / k T}$,

$N_{c}=N_{V}=2\left(m k T / 2 \pi \hbar^{2}\right)^{3 / 2}$,

$\left\{\begin{array}{l}m(T)=g_{c}^{2 / 3} K^{1 / 3} \cdot 0,048 \cdot m_{0} \sqrt{T / 300}, \\ g_{c}=4, K=m_{\|} / m_{\perp}=9(T \geq 300 \mathrm{~K}),\end{array}\right.$

$E_{g}(T)=0,217+4.5 \cdot 10^{-4}(T-77)(э \mathrm{~B})$,

$(T \geq 77 \mathrm{~K})$.

Here, $m_{0}$ is the free electron mass, and $K$ is the anisotropy constant of effective mass of electrons in conduction band and light holes in valence band.

$K_{F}^{\prime}$ was determined from equilibrium condition:

$K_{F}^{\prime}=K_{\mathrm{PbTe}}\left(T_{s}\right) K_{\mathrm{Pb}, V}^{\prime}\left(T_{s}\right) K_{\mathrm{Te}_{2}, V}^{\prime}\left(T_{s}\right) K_{i}^{-1}\left(T_{s}\right)$.

Assuming that in the studied pressure range vapour can be treated as ideal gas, for the quasi-equilibrium state of «vapour-condensate» system we get:

$K_{\mathrm{PbTe}}\left(T_{s}\right)=K_{\mathrm{PbTe}}\left(T_{e}\right) \cdot\left(\frac{T_{s}}{T_{e}}\right)^{3 / 2}$.

Other reaction constants used in this paper are listed in the Table; they were taken from previous results for $\mathrm{PbTe}$ crystals [2, 3].

4. Experimental and theoretical results of dependency of charge carrier and defect concentration in $\mathrm{PbTe}$ films on technological factors (i.e. partial $\mathrm{Te}_{2}$ vapour pressure $P_{\mathrm{Te}_{2}}$ and substrate temperature $T_{\mathrm{S}}$ ) in the hot-wall method are presented in Fig. 1, 2. It is clear (Fig. 1) that the increase in Te vapour pressure in precipitation region and increase in substrate temperature lead to the initial decrease in electron concentration. At some values $T_{s}^{*}$ and $P_{\mathrm{Te}_{2}}^{*}$, the inversion of conductivity type from $n$ to $p$ oc-

Table. Equilibrium constants $K=K_{0} \exp (-\Delta H / k T)$ of the quasi-chemical reactions.

\begin{tabular}{lcl}
\hline \hline Constants & $K_{0}$ & $\Delta H, \mathrm{eV}$ \\
\hline$K_{P b T e}, \mathrm{~Pa}^{3 / 2}$ & $1.4 \cdot 10^{18}$ & 3.51 \\
\hline$K_{P b, V}^{\prime}, \mathrm{cm}^{-6} \mathrm{~Pa}^{-1}$ & $5.5 \cdot 10^{30}$ & -1.01 \\
\hline$K_{T e_{2}, V}^{\prime \prime}, \mathrm{cm}^{-6} \mathrm{~Pa}^{-1 / 2}$ & $1.2 \cdot 10^{38}$ & 0.25 \\
\hline$K_{P b, V}^{\prime \prime}, \mathrm{cm}^{-9} \mathrm{~Pa}^{-1}$ & $1.2 \cdot 10^{51}$ & -0.87 \\
\hline$K_{T e_{2}, V}^{\prime \prime}, \mathrm{cm}^{-9} \mathrm{~Pa}^{-1 / 2}$ & $1.5 \cdot 10^{59}$ & 0.39 \\
\hline$K_{a}, K_{b}, \mathrm{~cm}^{-3}$ & $6.5 \cdot 10^{20}$ & 0.14 \\
\hline
\end{tabular}




\section{D.M. Freik et al.: Crystallochemistry of defects in lead telluride films}
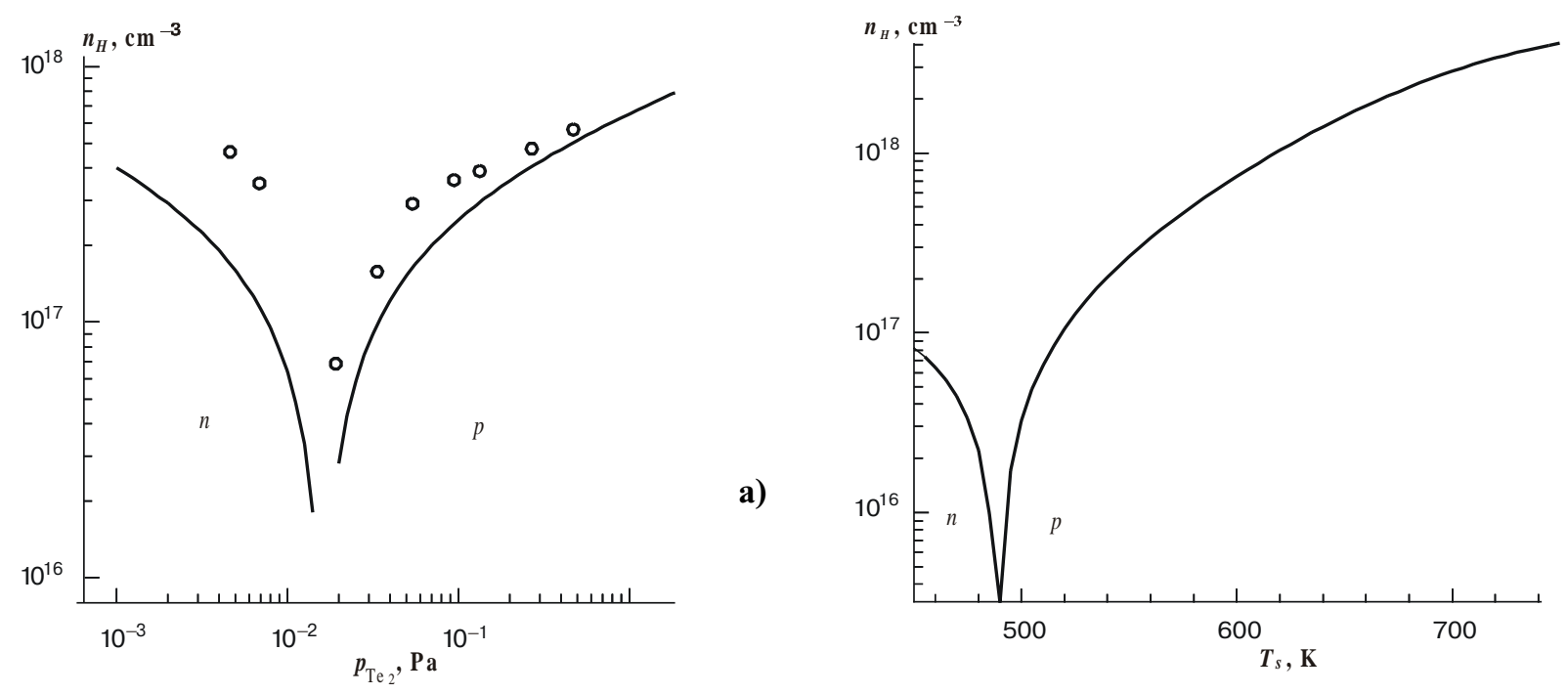

b)

Fig. 1. Dependence of carrier concentration in $\mathrm{PbTe}$ films on:

(a) - the partial pressure of tellurim vapour $\left(T_{s}=653 \mathrm{~K}, T_{e}=833 \mathrm{~K}\right)[12]$;

(b) - the substrate temperature $\left(P_{\mathrm{Te}_{2}}=1.65 \cdot 10^{-2} \mathrm{~Pa}, T_{e}=720 \mathrm{~K}\right)$; the substrates are splinters of (111) $\mathrm{BaF}_{2}$; the solid lines are calculated curves according to (16), (17).
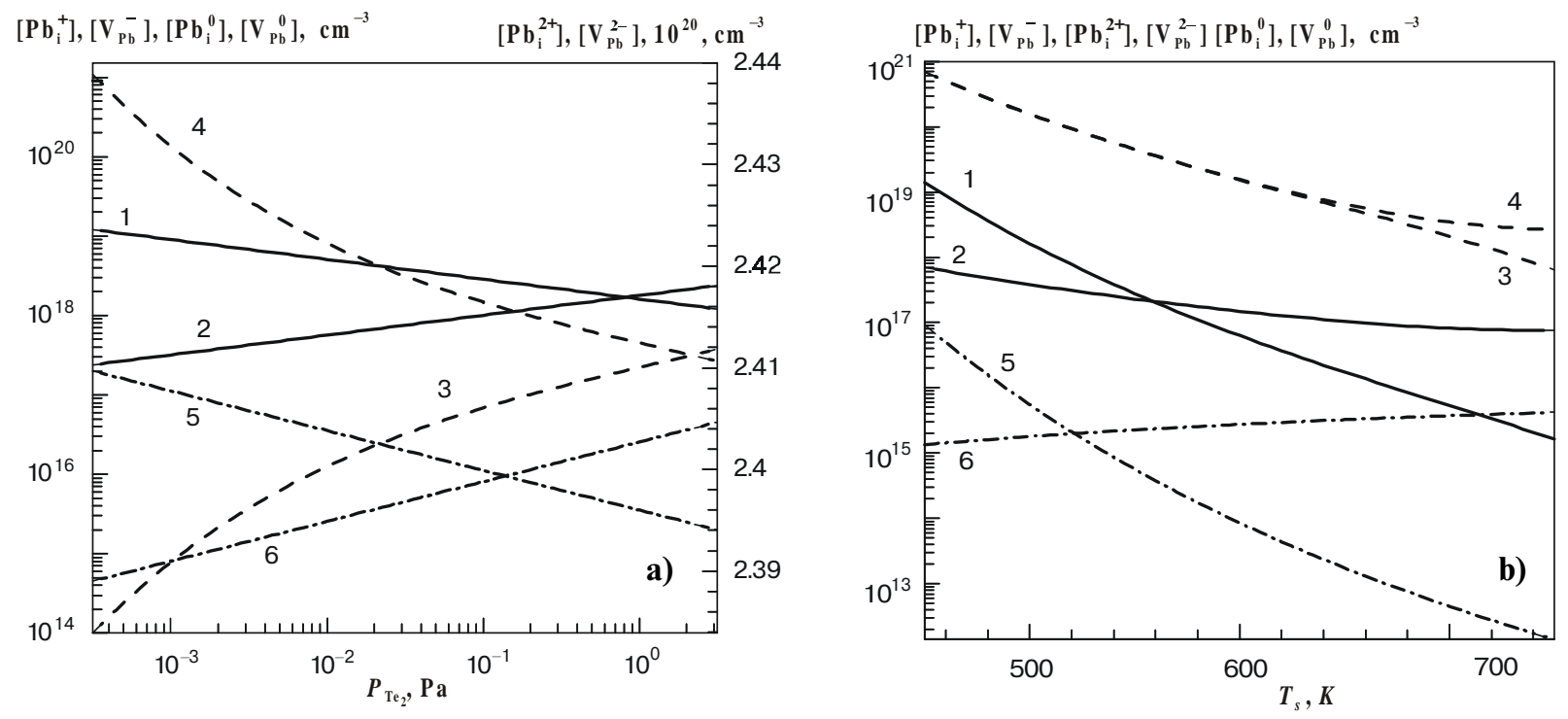

Fig. 2. Dependence of calculated concentration of defects according to (18) in PbTe films on:

(a) - the partial pressure of tellurim vapour $\left(T_{s}=653 \mathrm{~K}, T_{e}=833 \mathrm{~K}\right)$;

(b) - the substrate temperature $\left(P_{\mathrm{Te}_{2}}=1.65 \cdot 10^{-2} \mathrm{~Pa}, T_{e}=720 \mathrm{~K}\right)$.

The solid lines are singly charged defects $\left[\mathrm{Pb}_{i}^{+}\right](1)$ and $\left[V_{\mathrm{Pb}}^{-}\right](2)$, the dotted lines are doubly charged $\operatorname{defects}\left[\mathrm{Pb}_{i}^{2+}\right](3)$ and $\left[V_{\mathrm{Pb}}^{2-}\right]$ (4), the stroke-dotted lines are the electroneutral defects $\left[\mathrm{Pb}_{i}^{0}\right]$ (5) and $\left[V_{\mathrm{Pb}}^{0}\right]$ (6). 


\section{D.M. Freik et al.: Crystallochemistry of defects in lead telluride films}

curs, followed by the further increase in hole concentration. It is worth mentioning that this change in charge carrier concentration (Fig. 1) is accompanied by the change of concentration of vacancies and interstitial lead atoms (Fig. 2). With the increase in $T_{\mathrm{s}}$ and $P_{\mathrm{Te}_{2}}$, concentration of singly charged lead vacancies increases (Fig. 2, curve 1), and the concentration of singly ionized interstitial lead atoms decreases (Fig. 2, curve 2).

Doubly charged defects appear to be strongly compensated. According to our calculation (Fig. 2, curves 3, $4)$, their concentration is rather high and amounts to $\sim 10^{20} \mathrm{~cm}^{-3}$ at $T_{S}=653 \mathrm{~K}$. This is two orders of magnitude higher than the concentration of singly ionized defects $\left(\sim 10^{18} \mathrm{~cm}^{-3}\right.$, Fig. 2$)$, which is in turn exceeds the concentration of neutral defects (Fig. 2, curves 5,6). This is in full agreement with our assumption about the predominance of the multiply charged vacancies and interstitial atoms that can be in different charge states in the presence of sufficiently shallow acceptor and donor levels [13]. Due to strong but incomplete compensation of the doubly charged defects (Fig. 2, curves 3,4), singly charged defects play comparably important role in changing charge carrier concentration in $\mathrm{PbTe}$ films.

Thus, the crystal-chemical calculation of equilibrium of intrinsic atomic defects in cation sublattice of PbTe films shows that the assumption of simultaneous existence of singly and doubly charged defects is most reasonable and is in agreement with the experimental data.

\section{References}

1. N.Ch. Abrikosov, L.E. Shelimova, Semiconductor materials based on $A^{I V} B^{V I}$ compounds. Nauka, Moscow (1975) (in Russian).

2. V.P. Zlomanov, P-T-X-diagrams of two-component systems. MGU, Moscow (1980) (in Russian).

3. A.M. Gas'kov, V.P. Zlomanov, A.V. Novoselova, Homogeneity region of lead telluride // Izv. AN SSSR, Neorgan. mater. 15 (8), pp. 1476-1478 (1979) (in Russian).

4. M. Schenk, H. Berger, A. Klinakov, Non-stoichiometry and point defects in PbTe // Cryst. Res. And Technol. 23 (1), pp. 77-84 (1988).

5. D.M. Zayachuk, V.A. Shenderovskii, Intrinsic defects and electronic processes in $\mathrm{A}^{\mathrm{IV}} \mathrm{B}^{\mathrm{VI}} / / U k r$. Fiz. Zh. 36 (11), pp. 1692-1713 (1991) (in Ukrainian).

6. H. Heinrich, Defects in IV-VI compounds // Lect. Notes. 133, pp. 407-426 (1980).

7. D.M. Freik, Ya.P. Saliy, I.M. Lishchinsky, The properties of $\mathrm{PbTe}$ thin films and quasi-chemical modelling of vapor-phase epitaxy // Zh. Fiz. Chim. 71 (12), pp. 2135-2137 (1997) (in Russian).

8. Ya.L. Charif, I.Ch. Avetisov, T.V. Zaychuk, P.V. Kovtunenko, Ionized and electroneutral intrinsic point defects in $\mathrm{PbTe}$ and PbSe // Izv. AN SSSR, Neorgan. mater. 25 (4), pp. 567570 (1989) (in Russian).

9. D.M. Freik, M.A. Galushchak and L.I. Mezhylovskaya, Physics and technology of semiconductor films. Vyshcha shkola, Lvov (1988) (in Russian).

10. F.A. Kröger. The chemistry of imperfect crystals. Mir, Moscow (1969) (in Russian).

11. T.S. Moss, G.J. Burrel, B. Ellis, Semiconductor optoelecronics. Mir, Moscow (1976) (in Russian).

12. A. Lopez-Otero. The use of a phase diagram as a guide for the growth of PbTe films // Appl. Phys. Lett. 26 (8). pp. 470472 (1975).

13. V.L.Vinetskii, G.A.Cholodar. Statistical interaction of electrons and defects in semiconductors. Naukova dumka, Kiev (1969). 